\title{
Primary Exploration and Pre-Experimental Designs on the Measurement of Cognitive load as well as Statistics-Graph Reading Comprehension in Mathematics
}

\author{
Tsai-Wen Lin \\ National Normal Taiwan University, Taipei, Taiwan
}

\begin{abstract}
Graphic reading comprehension composes of the complicated mental process which differs from that involved within the text reading comprehension. Graphic reading comprehension, theoretically speaking, contains three pivotal phases: encoding, translating and identifying. Hence, the article aims to apply the modeling of graphic reading comprehension for exploring and framing the primary model of the mental contemplate concerning statistic-graph reading comprehension in mathematics. Furthermore, experimental designs will be contended in this study and criteria of eyetracking based on cognitive loads will be regarded as the evaluated units with a view to testifying the hypothetic mental model in the future research.
\end{abstract}

\section{Introduction}

Except general counting, mathematic thinking and analytic proficiency, the application of probability, space and graphs, quantitative reasoning, and the more difficult subjects inclusive of algebra or geometry are also involved within the field of mathematical assessment. For publics, on the other hand, they need to cope with versatile mathematical issues, such as statistic graphs or graphic information, which signifies that it is essential for the public to comprehend the statistic graphs with a view to dealing with mathematical problems in their daily life (Hung, Hsiao and Lin [6]). Furthermore, all too often, people contact several walks of information shown in a statistical way (numbers, graphs, etc.) via mass media; hence, to cultivate the proficiency for comprehending the statistical data included in the newspaper, magazines, advertisements, and TVprograms has turn into the basic statistical literature for the public (Chen [2]). Accordingly, as far as reading comprehension of statistical graphs is regarded as one of pivotal categories involved in the mathematical comprehension as well as assessment, and as the significant ability for doing mathematical problem-solving in the daily life.

Statistic-graph learning is one of mathematical concepts (Chou [4]) and the reading comprehension of learning process, the complete and specific reading process, is also contained in that of the mathematical texts. Moreover, perception and cognition of linguistic symbols, inclusive of words, signals, jargons, formula, and graphs, adaptation, accommodations are concerned as the mental functions within the reading comprehension as well as memorizing (Chin and Chiu [3]).In other words, readers or learners are able to come by the represented contents of the mass mathematical information in a verbal way via graphic representations, which signifies the importance of statistical graphs. Although they are shown prevailingly in the textbooks or mass reading materials for the public, it is often the case with the readers to get astray in the statistics-graph labyrinths (Shah, Mayer and Hegarty [11]). Additionally, Pinker [10] contended that the specificity of statistical graphs could have the effects on the proficiency of reading comprehension as appears the significance of the influential factors resulting in the disadvantaged statistics-graph reading comprehension.

Visual description and graphic schema are contained in the statistic-graph reading process. The former one explains that stimuli of figures encoded physically can be restricted by the limited capacity of short-term visual processing; the later one, yet, contends that how individuals form the encoded representations into proper mathematical scalar graphs (Pinker [10]). Discussed in detail, no less pattern recognition than integration are included 2in the executive process ; the one consists of encoding and translating the quantitative and qualitative into graphs and the other is composed of the connection linking the postulated referents according to the interpretative contents and graphs (Carpenter and Shah [1]). Based on the link of information process model and statistic-graph reading comprehension, the researcher integrated these dimensions to analyze readers' combination of graphic information.

On the other hand, in order to explore the mental functions within the process of statistic-graph reading via outer behaviors and physical performances, applied methods in the researches of statistic-graph reading are composed of protocol, eye-movement, and tests of reading comprehension 
(Carpenter and Shah [1]; Shah, Mayer and Hegarty [11]; Shah and Hoeffner [12]).

The article aims both to integrate the information process model and the fundamental frame of statistics-graph reading and make the initial exploration of the mental works involved in the process of statistics-graph reading. Furthermore, the research questions are shown as follows. What is the overlapping function between information process model and statistic-graph reading comprehension? What do the criteria of eye-movement make difference as the participants reading various kinds of statistical graphs?

As far as the criteria are concerned, they will be applied in accordance with cognitive theory. Sweller [14] contended the cognitive load theory, consisting of extraneous cognitive load, intrinsic cognitive load, and germane cognitive load. Extraneous cognitive load is influenced by the outer information; intrinsic cognitive load is affected by inner elements interactivity; germane cognitive load, however, accompanies with intrinsic cognitive load. However, the measurement of cognitive loads manipulated the following approaches: subjective techniques, physiology techniques, and task-and performance-based techniques. Majority of measurement applied to measure cognitive loads on educational issue is scaling or protocol analysis; yet, some confounding problems are involved in the scaling method. Hence, one of the preliminaries of this plan is to figure the precise criteria for measuring the cognitive loads when participants reading statics-graph.

\section{Literature Review}

\subsection{Statistic-graph reading involved in Mathematical Education}

Statistical graphs function as the instrument to present the raw data in a simpler and integrative way so that to read as well as to speak out the content of statistical graphs and to make statistical graphs are competency indicators (Ministry of Education). Via the outcomes of performed by Taiwan elementary and high school students shown in 2009's PISA, eighth-grade students' deficit ability of reading and comprehending statistical graphs was unveiled. The unfamiliarity with item curves, the deficit manipulation of mathematical professional skills in daily life and difficulties in statistic-graph comprehension were concerned as the postulated causes (Li, Wang, Li, Su and Chen [8]). Precise comprehension of statistical graphs and functional application signify the essence for statistic-graph learning in Mathematical Education.

The main factors causing pupils' misreading statistical graphs contain graphic visual representations, readers' knowledge of graphs, and their expectations for the contents involved in the graphs (Shah and Hoeffner [12]). Additionally, Kelly [7] also contended that both the destinations including of graph-user and characteristics of tasks including graphic perception, visual encoding, and types of analytic tasks and so on can be the pivotal effects on graph reading comprehension.

Briefly speaking, statistic-graph reading comprehension can be affected by the interaction intertwined with characteristics of graphs regarded as stimuli information and reader's cognition concerned as the ability to execute the mental activity when individuals reading the statistical graphs.

\subsection{Exploration of information process model and statistic-graph reading theory}

Atkinson and Shiffrin contended the information process model, consisting of sensory memory, shortterm memory, and long-term memory in which domain registers belong to different stimuli forms with a view to registering sensory memory. Furthermore, registered information will be controlled and executed in working memory or retrieve from long-term memory; namely, the procedure inclusive of decoding, rehearsing, encoding, deciding, and retrieving for dealing with new information occurs in working memory. Sternberg [13] nevertheless presented the executing model composed of both the general domain and the specific domain. The former one elucidates the responses of inputs and the outputs via vocabulary analytic task experiment; the later one emphasizes the change of specific-domain development (Sternberg [13]). Due to the limited explanation of information process model for human complex mental activity and restricted with parallel processing as well as context effect, Neisser [9] held the revised model via correcting the classic information process model in which sensory memory, working memory and long-term memory make a dual executive processing when individuals receiving stimuli. Accordingly, the center of information process model lies in the process as individuals receiving stimuli, two facets of shortterm memory and long-term memory with versatile cognitive action.

On account of the complexity of statistic-graph reading process and the familiarity of its procedure with information process model, this article aims to integrate the perspectives of models mentioned above. In the first place, graph theory mentioned in the article contended by Pinker [10] in accordance with characteristics of graphs contains two major facets. Visual descriptions encoded physically and graphic schema forming the mathematical scalar graphs according to the former ones involved in the graphic representation function when individuals 
elucidate the contents of graphs. Couples of dimensions contained in the graph-reading process consist of matching (comparison with memory schema of visual descriptions and visual backgrounds), message assembly (translation of visual information and conceptual one), interrogation (retrieving former information), and inferential. As a result, the holistic process can be divided into three core phases: to encode stimuli represented visually and to discern imperative objectives, to connect visual objectives and relationships contained in them, and to numberize the referential concepts (Shah and Hoeffner [12]). To sum up, it makes difference between information process model and statisticgraph reading for the span of stimuli inputs; postulated elaborately, the fundamental frame of statistic-graph reading process means information process model, yet graph-reading can be regarded as information process model subordinated to the specific domain. Thereby, not all stimuli information will store into long-term memory, but applied to make reasoning.

\subsection{Cognitive load theory and the measurement}

Sweller (1998) contended cognitive load theory on the basis of information process and working memory so that three kinds of cognitive loads was interpreted and hypothesized. Furthermore, seven effects, affecting the occurrence of these cognitive loads, was held, inclusive of goal free effect, worked example effect, split-attention effect, completion problem effect, modality effect, redundancy effect, and variability effect. Hence, the manipulation of experimental designs will formulated according to the effects mentioned above so as to acquire the correct criteria to measure the cognitive loads.

\subsection{Researches concerning statistic-graph reading}

Carpenter and Shah [1] manipulated the eye tracker to discover the eye tracks of pattern recognition and integration within the procedure of encoding, translating, and identifying. The material for the experiment was the graph with the five divided regions inclusive of the title, $\mathrm{x}$-axis, $\mathrm{y}$-axis, z-criteria, and the center of graphs, and the eye tracking showed the mental actions occurring during different phases. The collected quantitative data with gaze numbers and time were functioned as the comparative criteria for experiment group as well as control group; the qualitative data was the selfprotocol. The followings were the findings. Integration process model signified the connection of graphs and information concerning graphs. Comprehension process showed the increase of the quantitative data. Complexity, numbers and familiarity with information about the graphs of materials had effects on the individual continued mental efforts to realize the experiment materials. For another thing, Shah, Mayer and Hegarty [11] applied the experiment materials with "fewer inferential contents but more connection of patterns" and "wrong inference within the complicated graphreading process". Participants were 28 American College students, divided into experiment group with 16 ones and control group with 12 ones. The unveiled findings compiled via self-protocol during the experiment process were reported as follows. Fewer inferential contents but more connection of patterns counted for readers. Chunks of reading information fostered readers' comprehension for the contents of graphs.

In accordance with results from literature review, gaze numbers and time can be regarded as the criteria for the definitions of different stages within mental actions when individuals reading statistical graphs. However, it's still essential for researchers to explore the definitive criteria for detail mental actions. Accordingly, the researcher has planned to make experiment designs to confirm the precise relationship between statistic-graph reading and working memory.

\section{Research Rationale}

\subsection{Method}

\section{Experiment 1}

Within group and between group will be manipulated for formulating the experiments to measure the eye-tracking when participants reading statistics-graph. Participants in within group view the materials for measuring the intrinsic cognitive load and that in between group read the materials concerning measuring extraneous cognitive load. Data analysis will aim to come by the discern criteria for different cognitive loads so as to be applied to acquire the cognitive loads in the procedure of statistics-graph reading.

\section{Experiment 2}

Quasi-experiment (2*4) and within subject design will be planned to be applied in this article. Population will be the students not majoring in mathematics, psychology and statistics of a certain university in Taiwan. That is, convenient sampling will be used in the article. Then, participants will read the original and manipulated materials. Furthermore, materials will be composed of four kinds of graphs: bar, line, pie, and curve.

\section{Procedure}

Both in these two experiments, first, researcher will ask participants for reading out the pseudo 
materials with an eye to confirming their reading skills for statistical graphs. Participants will need to read all graphs and make self protocols.

\section{Instrument}

Eye link1000 will be manipulated in the experiment to collect the eye tracks of participants. The test of reading comprehension made by the researcher will be taken as the way to realize participants' understandings of the texts.

\section{Analysis of Findings}

Fixation time, gaze numbers and saccade-length will be regarded as the criteria. Two-factor multiple variances will be applied to analyze the raw data from eye tracks.

\section{Conclusion}

The integrated model in accordance with literature review

\section{Expected results}

The difference of the averages of fixation time and gaze number for the separate areas within graphs will be significant. It is also the same expected result with that of different sorts of graphs.

\section{Future issues}

Cognitive load theory can be integrated to explore the more precise relationship between information process model and graph theory, which were to establish the physical criteria for cognitive load. On the other hand, the statistic-graph reading model is able to be applied as one of the criteria for compiling mathematical textbooks and designs of statistical graphs shown to the public in daily life. Accordingly, physical criteria of cognitive load will be erected and the evaluation of statistics-graph involved in mathematical textbooks will be followed through.

\section{Reference}

[1] Carpenter, P. A. and Shah, P., (1998). A model of the perceptual and conceptual processes in graph comprehension. Journal of Experimental Psychology: Applied, 4(2), 75-100. Retrieved from EBSCOhost.

[2] Chen, H. M., (2006). Connotation and teaching rationale for statistical curriculum in elementary school. Science Education Monthly, 287,2-12.

[3] Chin, L. H., Chiu, S. C., (2004).The development of a model for mathematics text reading comprehension: angel unit in the mathematics as an example.Special education,12,99-121.
[4] Chou, Y. H., (2006). Reviewing math literacy and education by means of PISA. Science Education Monthly,293, 2-21.

[5] Friel, S. N., Curcio, F. R., (2001). Making sense of graphs: critical factors influencing comprehension and Instructional Implications. Journal for Research in Mathematics Education,2(32), 124-158.

[6] Hung, P. H., Hsiao, C. W., Lin, S. W., (2009). Implications of cognitive component analysis on PISA mathematical literacy assessment for supplemental instruction design. Curriculum and Teaching,13(1),47-66.

[7] Kelly, J. D. (1993). The Effects of Display Format and Data Density on Time Spent Reading Statistics in Text, Tables, and Graphs. Journalism Quarterly, 70(1), 140-49. Retrieved from EBSCOhost.

[8]Li, Y. S., Wang, M.J.,Li, M.S,Su, Y.W., Chen,Y.J. (2009), Analysis and application of high and elementary school students' mathematical competency in Taiwan. published in "Fundamental researched of curriculum for high and elementary school” conference.

[9] Neisser, U., (1976).Cognition and reality. San Francisco: Freeman.

[10] Pinker, S. (1990), A theory of graph comprehension. In R. Freedle (Ed.), Artificial intelligence and the future of testing (pp. 73-126). Hillsdale, NJ:Erlbaum.

[11] Shah, P., Mayer, R. E., and Hegarty, M., (1999).Graphs as aids to knowledge construction: signaling techniques for guiding the process of graph comprehension. Journal of Educational Psychology, 4(91), 690-702.

[12] Shah, P.\& Hoeffner, J., (2002). Review of graph comprehension research: implications for instruction. Educational Psychology Review, 1(14), 47-69.

[13] Sternberg, R.J., (2005).Cognitive Psychology. New York: Publish.

[14] Sweller, J., (1988). Cognitive load during problem solving: Effect on learning. Cognitive Science, 12, 257285. 